\title{
Intensifiers and image schemas: Schema type determines intensifier type Kevin King*
}

\begin{abstract}
This paper develops a theory whereby the type of intensifier that a source word grammaticalizes as is determined by the semantics of the source word. Three intensifiers (way, thoroughly, and overly) are looked at in-depth and it is shown that their semantics as intensifiers follow from certain features, namely path and endpoint, of the image schemas depicting the meanings of their source words. It is possible that this theory can be extended to all intensifiers, especially given that many intensifiers grammaticalize out of source words that have spatial interpretations.
\end{abstract}

Keywords. intensifiers; image schemas; semantics

1. Introduction. Reference grammars of English (Quirk et al. 1985, Huddleston \& Pullum 2002) have categorized intensifiers according to the semantic function they serve, and more than a century of research on intensifiers in English (e.g. Stoffel 1901, Kirchner 1955, Partington 1993, Lorenz 2002, Ito \& Tagliamonte 2003, Claudi 2006, Macaulay 2006, Mendez-Naya 2008, Gonzalez-Diaz 2008) has established that intensifiers grammaticalize out of adjectives and adverbs. Gradually, the semantics of source words fade out until they acquire general meanings of degree. Recent research, often bolstered by the availability of large searchable electronic corpora, has established the exact course of semantic change taken by individual source words as they grammaticalized as intensifiers.

What is missing is a general theory of intensifiers that can explain why a given source word grammaticalizes as one type of intensifier rather another. In this paper, I lay the groundwork of such a theory. Briefly, I claim that the semantics of source words, which I depict using image schemas, determine the type of intensifier those source words grammaticalize as. This theory necessarily assumes that the concepts of space and degree are closely related, an assumption that finds support in cognitive science and typological literature.

2. Types of Intensifiers. Reference grammars of English offer differing definitions of "intensifier," as well as differing categories into which intensfiers may be placed. Quirk et al. (1985) refer to all degree adverbs as intensifiers, which can be divided into Amplifiers (intensifiers that scale upwards) and Downtoners (intensifiers that scale downwards). These two categories are further broken down; Boosters and Maximizers are types of Amplifiers, while Approximators, Compromisers, Diminishers, and Minimizers are types of Downtoners. Quirk et al.'s taxonomy is broken down in Table 1 .

\begin{tabular}{l|l|l|l} 
Amplifiers & \multicolumn{1}{l|}{ Examples } & Downtoners & \multicolumn{1}{l}{ Examples } \\
\hline Boosters & She's a really nice person. & Approximators & I'm almost full. \\
Maximizers & My shoes were completely soaked & Compromizers & She's more or less a genius. \\
& & Diminishers & I feel somewhat sick. \\
& Minimizers & He's hardly qualified. \\
\hline
\end{tabular}

Table 1: Quirk et al.'s (1985) taxonomy of intensifiers

* Thank you to Patrick Farrell for consultation and feedback concerning this paper. Author: Kevin King, UC Davis (kerking@ucdavis.edu). 
Bolinger's (1972) definition of intensifiers is largely the same as that of Quirk et al. Bolinger calls any degree adverb an intensifier, but breaks the category down a bit differently into Boosters (Quirk et al.'s Amplifiers), Compromisers (Quirk et al.'s Approximators and Compromisers), Diminishers, and Minimizers.

Huddleston and Pullum (2002), on the other hand, define intensifiers as specifically those degree adverbs that scale upwards, corresponding to Quirk et al.'s Amplifiers. This is by and large the definition adopted by researchers working on intensifier grammaticalization. Claudi (2006: 352), for example, remarks that it is "hard to understand why an element called 'intensifier' should express the bare opposite of intensification, namely diminishing or minimizing...or something like a compromise." In keeping with this tradition in the diachronic literature, the focus in this paper is only those degree adverbs that scale upwards. Hereafter, the word "intensifier" refers to degree adverbs that scale upwards, corresponding to Amplifiers in Quirk et al. and Boosters in Bolinger.

Still, it is important to recognize, as Quirk et al. do, that the category of intensifiers is comprises several sub-types that have different semantic functions. Quirk et al. break down intensifiers into Boosters and Maximizers, which are degree adverbs that scale upward to a very high and a maximal degree, respectively. In addition to these types, I add a third: Excessivizers, or intensifiers that scale upward beyond a desired or expected maximum.

\section{Boosters Jane is a very good singer. \\ Maximizers I'm thoroughly stuffed. \\ Excessivizers This soup is overly salted.}

Table 2: Types and examples of intensifiers examined in this paper

Below I will develop a theory explaining why source words grammaticalize as Boosters, Maximizers, and Excessivizers. This theory relies on image schemas, a technique developed in cognitive semantics for describing the senses of (especially) words that have spatial interpretations. Before developing these ideas, it is first necessary to briefly explain image schemas.

3. Image Schemas. Image schemas have proven to be a useful way to capture the various interrelated senses of prepositions (Brugman 1983, Lakoff 1987, Tyler \& Adams 2003), as well as to describe abstract concepts such as causation and obligation (Johnson 1987).

A well-known example is Brugman's classification of the different senses of over. Each different sense corresponds to a different image schema, and the image schemas are linked to one another according to their similarities. Out of these linkages, a single central sense of over can be identified, to which all the others are related and from which all the others are derived. Tyler and Adams picked up this technique and applied it to many more English prepositions.

Typical image schemas involve the spatial relationship between a landmark (LM) and a trajector (TR). For example, an image-schematic depiction of the sense of over in the sentence The picture hung over the mantle would include a LM corresponding to the mantle, with a TR corresponding to the picture positioned above it. Other schemas involve additional features; the two such features relevant to this paper are path and endpoint. In the schema for the sense of over in The plane flew over the barn, the TR corresponding to the plane moves along a path that takes it into and then out of a position that is directly above the LM corresponding to the barn. The path in the schema for the sense of over in the sentence The post office is over the hill contains an endpoint; that is, there is a path that begins at a point on one side of the hill and ends 
at a point on the other side of it where the post office (TR) is located. A schema for yet another sense of over contains a (desired) endpoint, but the path does not stop there and instead continues past it. Thus, in the schema for over in The water swelled over the dam, there is an endpoint, corresponding to the highest part of the LM (the dam), and the TR (the water) moves beyond it.

Thus, in addition to image schemas in which the LM and TR are stationary, there are Path (The plane flew over the barn), Endpoint-Focus (The post office is over the hill), and Excess (The water swelled over the dam) schemas. In what follows, I will argue that intensifiers grammaticalize out of source words whose semantics are in some way based on these schemas, and that the type of intensifier a source word grammaticalizes as is predictable from the type of image schema the source word's semantics are based on. Specifically, I argue that Path, Endpoint-Focus, and Excess schemas correspond to Boosters, Maximizers, and Excessivizers, respectively.

The discussion to follow focuses on three intensifiers: way (source word: away), thoroughly (thorough/through), and overly (over). These examples were not picked at random; rather, they were chosen specifically because they are rather transparently related to source words that have spatial meanings. This makes them particularly suited to the image-schematic analysis presented here. However, this is not to say that this analysis is necessarily restricted to intensifiers whose source words have spatial interpretations. Rather, I assume that the semantics of degree are based on the semantics of space. This assumption finds support in the cognitive science and typological literature. Specifically, cognitive science research suggests that image schemas are incorporated into non-spatial, non-imageable types of knowledge (Mandler \& Cánovas 2014: 527), and research in typology shows that spatial words frequently grammaticalize as degree words and morphemes cross-linguistically (Fortuin 2013).

4. Way. According to Bolinger (1972) and Kirchner (1955), the intensifier way is descendent of away. This assumption is supported by corpus evidence, which shows that way was originally a phonologically reduced form of away.

(1) Mitchell, that letter DeWitt wrote you, has gone away — way down yonder. (GB, 1876)

(2) Such a lovely blue sky away, way up so high! (GB, 1888)

The semantics of away can be schematized using a LM, TR, and path (Figure 1). The TR is conceptualized as moving along a path that extends from LM to TR and beyond. This may go against one's intuitions: While some uses of away refer to entities moving along a path, others do not. Thus, in the sentence The waiter backed away from the cockroach, the TR representing the waiter is moving along a path away from the LM the cockroach. But in Judy is away from home, the TR Judy may or may not be stationary. Nevertheless, in such cases the TR must be conceptualized as having moved along a path away from the LM, and moreover the two must be conceptualized as having once occupied the same point in space at a previous time. Otherwise, accounting for the unacceptability of *Arizona is away from California would be impossible; if all that is required of away is that there be some spatial separation between LM and TR, then such sentences should be acceptable. It is only because of the hearer's world knowledge that Arizona and California have never occupied the same point in space before becoming separated that such a sentence proves problematic.

A simple test using sentence frames can show whether an intensifier is a Booster, Maximizer, or Excessivizer.

(3) a. A and B are x different, but not very different.

b. A and B are $\mathrm{x}$ different, but not completely different. 
c. A and B are $\mathrm{x}$ different, but not excessively different.

In (3a-c), $x$ can stand for any intensifier. If the sentence formed using the frame in $(3 \mathrm{a})$ is unacceptable, but those formed using ( $3 b-c)$ are acceptable, then the intensifier is a Booster. If

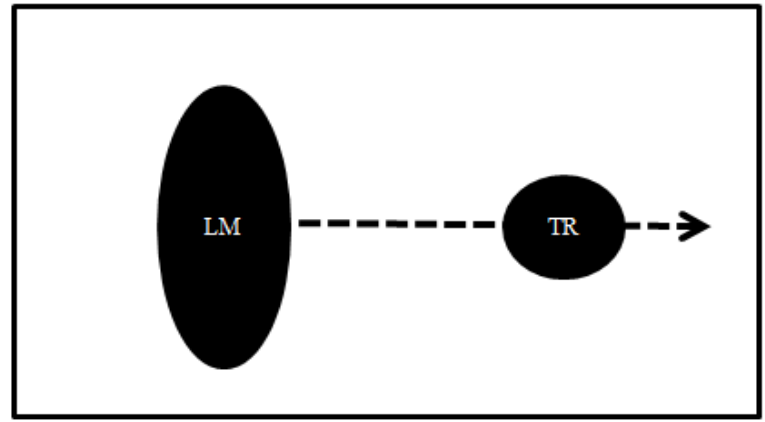

Figure 1. Image schema for away

(3b) is unacceptable and (3c) is acceptable (leaving aside (3a), as it is neither necessary nor sufficient for determining whether a given intensifier is a Maximizer), then the intensifier is a Maximizer. And if at least (3c) is unacceptable, then the intensifier is an Excessivizer.

(4) a. * A and B are way different, but not very different.

b. A and B are way different, but not completely different.

c. A and B are way different, but not excessively different.

It is therefore clear that way is a Booster.

If, as Mandler \& Cánovas (2014) claim, the semantics of degree are mapped onto the semantics of distance, then it is unsurprising that way should be a Booster. The image schema for away includes a path but no goal or endpoint; that is, there is no minimum distance that must exist between the TR and LM in order for the TR to be considered away from the LM. When away grammaticalized as way, this schema remained largely the same, but applied to degree rather than distance. The path, then, is reinterpreted as a degree scale, with an increase in distance corresponding to a rise in degree.

5. Thoroughly. The intensifier thoroughly is a Maximizer; it boosts upwards to a maximal degree. This is clearly seen by applying the sentence frame test.

(5) a. * A and B are thoroughly different, but not very different.

b. * A and B are thoroughly different, but not completely different.

c. A and B are thoroughly different, but not excessively different.

$(5 \mathrm{c})$ is acceptable because there is nothing about being thoroughly different that expresses a notion of excessiveness or going beyond a desired maximum. It is frequently the case that two things that are thoroughly different complement each other perfectly - like hot food with a cold beverage.

Thoroughly is an adverb related to the preposition through, both of which are ultimately descendent of Old English poru. When stressed, poru was pronounced as two syllables (giving rise to thorough), and when unstressed it was pronounced as a single syllable (like through). This split into two forms with specialized meanings; through became a preposition with a primarily spatial meaning, while thorough (and then thoroughly) was an adverb carrying a sense of completeness.

Understanding the relationship between the original spatial meaning and the later meaning of completeness is facilitated by turning to the image schema for through (Figure 2). In this 
image schema, a TR moves along a path that passes into a bounded LM and then out of it on the opposite side. Crucially, this schema has endpoint focus; that is, the TR must at the very least exit the LM after passing into it. This is evident by the unacceptability of sentences such as *The truck drove through the tunnel, and it is still in the tunnel.

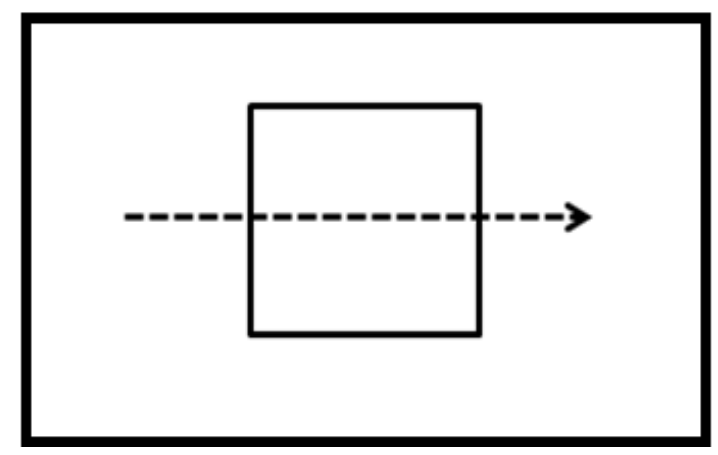

Figure 2. Image schema for through (Adapted from Tyler \& Evans 2003)

As the spatial semantics were bleached out of thorough, this image schema was repurposed as a degree scale, with the endpoint corresponding to a maximum. Whereas a truck must reach the endpoint of a tunnel in order to have gone through the tunnel, a property must be expressed to a maximal degree in order for that property to be expressed thoroughly.

6. Overly. The last intensifier to be discussed in depth is overly. Overly is an Excessivizer; it boosts beyond a desired or expected maximum. The sentence frame test shows this.

(6) a. ? A and B are overly different, but not very different.

b. ? A and B are overly different, but not completely different.

c. * A and B are overly different, but not excessively different.

(6c) is clearly unacceptable; if two things are overly different, then they are necessarily also excessively different. In the cases of (6a-b), it is possible to image scenarios where two items may be overly different but not completely different or even very different. For example, there may be a situation in which a houseguest has broken their host's lamp, unbeknownst to the host, and needs to find a replacement before the host finds out. The only suitable replacement lamp would be one that is indistinguishable from the original. If a candidate is identical in every way but one, it will be overly different, even though it is not completely different or even very different from the original. For this reason, these two sentences are rated as questionable. Neverthless, it is only (6c) which is necessary for determining whether an intensifier is an Excessivizer.

Figuring out the image schema for over, the source word for overly, is more difficult than in the cases of way and thoroughly. This is because over is a highly polysemous word. Indeed, it was over's polysemy that launched image schema research on prepositions in the first place (Brugman, 1983). Tyler and Evans (2003) identify two senses of over that denote excess. These are the Above-and-Beyond sense (exemplified in (7) and schematized in Figure 3) and the Overand-Above sense (sentence (8) and Figure 4).

(7) The batter hit the ball over the outfield fence.

(8) The river swelled over its banks.

In the schema for the Above-and-Beyond sense of over, the TR begins at a point that is not above the LM and then moves along a path that takes it above the LM and beyond it without ever 
coming into contact with it. In the schema for the Over-and-Above sense, the LM is a container and the TR is an entity held by the LM. The volume of the TR becomes too much for the LM to hold, resulting in spillage.

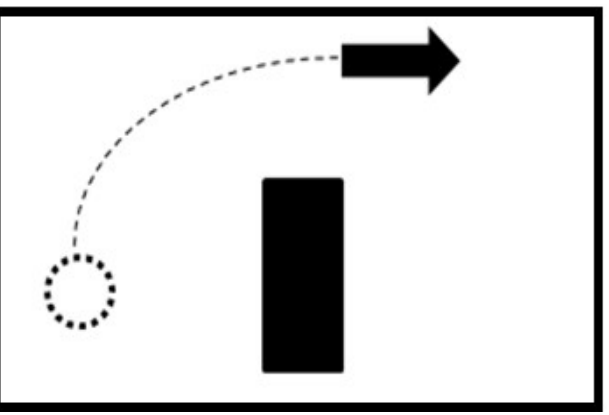

Figure 3. The Above-and-Beyond sense of over (adapted from Tyler \& Evans 2003)

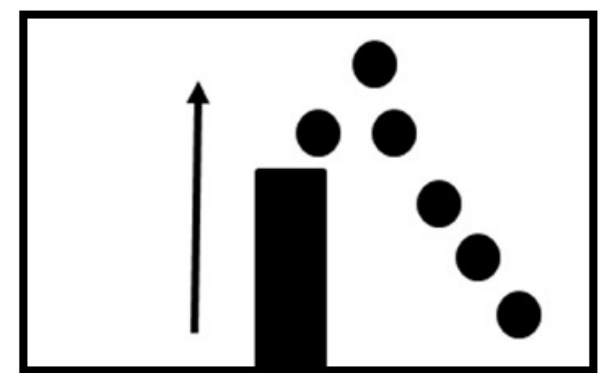

Figure 4. The Over-and-Above sense of over (adapted from Tyler \& Evans 2003)

It is difficult to decide which of these two senses is the source for the intensifier overly. Thankfully, these senses can actually be collapsed into a single sense using a technique employed by Tyler and Evans themselves when they reduced Lakoff's and Brugman's twentyfour senses of over down to fifteen. The technique is this: When an element in an image schema can be inferred from sentence context, it should be removed. For example, Lakoff and Brugman consider the two sentences in (9) to convey different senses of over, while Tyler and Evans consider them to be a single sense.

(9) a. The helicopter hovered over the ocean.

b. The bee hovered over the tulip.

According to Lakoff and Brugman, in (9a) the schema for over contains an extended LM whereas (9b) has a vertical one. But Tyler and Evans point out that the features of the LM are not contributed by over itself; rather, the extendedness or verticality of the LM are contributed by other words, specifically ocean and tulip.

This same line of thinking can be used to reduce Tyler and Evans' two Excess schemas down to one. In the Above-and-Beyond image schema, the LM is vertical and the TR moves along a path that takes it above and beyond the LM without touching it. In the schema for Overand-Above, the LM is a container and the TR is a fluid held by the LM which becomes too voluminous and overflows. These differences do not really come from unique senses of over, however; instead, they come from sentence context. In (7), the verticality of the LM is inferred from the word fence, and the lack of contact between TR and LM is inferred from basic world knowledge about typical baseball scenarios. Likewise, in (8), the fact that the LM is a container is inferred from banks, and the fluid nature of the TR is gotten from river and swelled.

Moreover, when a fluid overflows its container, it crucially flows over and above the walls of the container, and so the LM can be taken to be the vertical wall of the container rather than a 
container itself. Thus, the Above-and-Beyond and Over-and-Above senses can be reduced to a single sense, schematized in Figure 5.

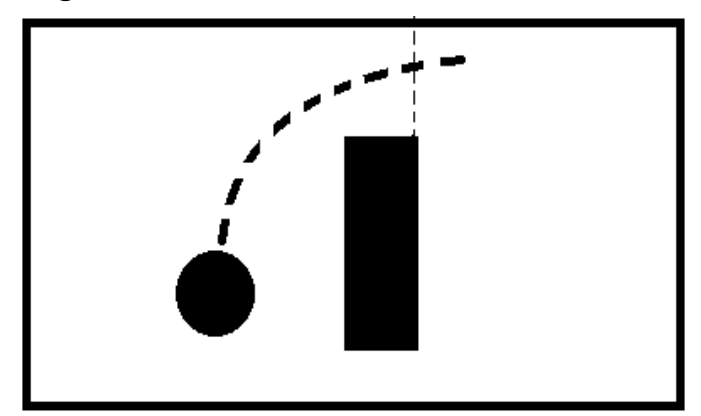

Figure 5. The Excess schema for over

In this image schema for the Excess sense of over, there is a TR that moves along a path that takes it higher than and across the top of the LM. This path has an endpoint, corresponding to the point where the TR crosses the vertical plane that comprises the exterior wall of the LM. But rather than stopping at the endpoint, the TR keeps moving past it, which results in overtaking or spillage, dependent upon context.

Either through semantic bleaching or by applying a conceptual metaphor (Lakoff 1987), this schema is reinterpreted to apply to degree rather than spatial relations. The endpoint corresponds to a desired maximum which, when surpassed, denotes excess. As the spatial character of the image schema is bleached out, so too do the spatial dimensions of its elements. It becomes irrelevant, for instance, whether the LM is vertical or not, and it likewise does not matter whether the path along which the TR travels goes up or not. All that matters in the end is that the path crosses the endpoint, denoting excess. In the end, then, the LM is either bleached out completely or it is conflated with the endpoint.

7. Conclusion. In this paper I have tried to fill a gap in the literature on intensifiers by outlining a general theory of their origins and grammaticalization. This theory utilizes image schemas as a convenient apparatus for demonstrating the relationship between source words and the intensifiers they later grammaticalize as. For the source meanings of the three intensifiers way, thoroughly, and overly, features of the image schemas are mapped onto spatial relations between entities; for the intensifier meanings, these same features are mapped onto the concept of degree.

An important takeaway of this theory is that the image schemas can be related to multiple domains of meaning. In the case of the intensifiers examined in this paper, the image schemas can apply either to space or to degree. But there is no reason to think that these would be the only two types of meaning to which such image schemas can apply. For instance, the most common and productive English intensifier, very, is descendant of a word meaning "truthful, actual," verray (cf. veracity). The present theory offers a way to explain the relationship between the source word and the Booster it grammaticalized as: The concept of truth is mapped onto a degree scale, such that there are "degrees of truth." Something that could be described at one time as verray would be at the upper end of this scale. Over time, as the semantics of veracity were bleached out of the word's meaning, the degree scale became the only relevant feature, and it became a Booster based on the same meaning depicted in the image schema for way.

When it comes to the numerous intensifiers whose source meanings are overtly spatial, the explanation is straightforward: Paths are reintepreted as degree scales, and endpoints as maximums. For example, the Maximizer roundly is based on the image schema for $(a)$ round. In 
order to travel (a)round some object, one must circumnavigate that object at least once, arriving back at one's starting position. This position is an endpoint, and when the schema is applied to degree, this endpoint becomes a maximum, resulting in a Maximizer interpretation.

It should be mentioned that the present paper is not the first attempt to come up with a general characterization of intensifer grammaticalization. Claudi (2006) and Lorenz (2002) both provide accounts that are meant to capture the general path along which words lose their rich semantics and acquire the general meaning attributed to intensifiers, but neither was able to go beyond a surface description of the phenomenon. Lorentz puts source words for intensifiers into five categories, and states that candidates will undergo semantic bleaching as they become intensifiers. Claudi offers a 5-stage cline of grammaticalization tracking the birth, flourishing, and death of intensifiers, but again there is no attempt at explaining why or how some words become one type of intensifier while others become another. This paper is not meant to contradict or displace either of these contributions. Rather, its purpose is to connect such accounts to the different categories of intensifiers recognized in the synchronic literature (Quirk et al. 1985, Huddleston \& Pullum 2002).

Further cross-linguistic work is needed to explore whether intensifiers and other degree words develop along the same lines described in this paper. Fortuin (2013) offers evidence that the results may be positive: In numerous languages, the concepts of excess and sufficiency are associated with path and goal. Thus, one would expect to find that path morphemes develop into elements that boost degree and endpoint-focus morphemes into elements that are Maximizers or Excessivizers, depending one whether the endpoint is reached or surpassed.

\section{References}

Bolinger, D. 1972. Degree Words. The Hague: Mouton.

Brugman, C. 1983. Story of over. Bloomington: Indiana University Linguistics Club.

Claudi, U. 2006. Intensifiers of adjectives in German. STUF, 59(4): 350-369.

Fortuin, E. 2013. The construction of excess and sufficiency from a crosslinguistic perspective. Linguistic Typology 17(1). 31-88.

Gonzalez-Diaz, V. 2008. Recent developments in English intensifiers: The case of very much. English Language and Linguistics, 12(2). 221-243. http://dx.doi.org/10.1017/S1360674308002608

GB = Davies, Mark. 2011-. Google Books Corpus. (Based on Google Books n-grams). Available online at http://googlebooks.byu.edu/.

Huddleston, R. \& Pullum, G. K. 2002. The Cambridge Grammar of the English Language. Cambridge, UK: Cambridge University Press.

Ito, R. \& Tagliamonte, S. 2003. Well weird, right dodgy, very strange, really cool: Layering and recycling in English intensifiers. Language in Society 32: 257-279. http://dx.doi.org/10.10170/S0047404503322055

Johnson, M. 1987. The Body in the Mind. Chicago: The University of Chicago Press.

Lakoff, G. 1987. Women, Fire, and Dangerous Things. Chicago: University of Chicago Press.

Kirchner, G. 1955. Gradadverbien: Restriktiva und Verwandtes im heutigen English (britisch und amerikanisch). Halle, Germany: Max Niemeyer Verlag.

Lorenz, G. G. 2002. Really worthwhile or not really significant? A corpus-based approach to the delexicalization and grammaticalization of intensifiers in Modern English. In Ilse Wischer \& Gabriele Diewald (eds.), New Reflections on Grammaticalization. 143-161. Amsterdam: John Benjamins Póublishing Company.

Macaulay, R. 2006. Pure grammaticalization: The development of a teenage intensifier. 
Language Variation and Change, 18. 267-283.

http://dx.doi.org/10.1017/S0954394506060133.

Mandler, Jean M. \& Cánovas, Cristóbal P. 2014. On defining image schemas. Language and Cognition 6. 510-532. http://dx.doi.org/10.1017/langcog.2014.14.

Mendez-Naya, B. 2008. On the history of downright. English Language and Linguistics, 12(2). 267-287. http://dx.doi.org/10.1017/S1360674308002621

Partington, A. 1993. Corpus evidence of language change: The case of the intensifier. In Mona Baker, Gill Francis \& Elena Tognini-Bonelli (eds.), Text and Technology: In Honour of John Sinclair. 177-192. Amsterdam: John Benjamins Publishing Company.

Quirk, R., Greenbaum, S., Leech, G., \& Svartvik, J. 1985. A Comprehensive Grammar of the English Language. New York: Longman.

Stoffel, C. 1901. Intensives and Downtoners: A Study in English Adverbs. Heidelberg: Carl Winter's Universitatsbuchhandlung.

Tyler, A. \& Evans, V. 2003. The Semantics of English Prepositions. New York: Cambridge University Press. 